\title{
Quantum entanglement and Hawking temperature
}

\author{
S. Santhosh Kumar ${ }^{\mathrm{a}}$, S. Shankaranarayanan ${ }^{\mathrm{b}}$ \\ School of Physics, Indian Institute of Science Education and Research Thiruvananthapuram (IISER-TVM), \\ Thiruvananthapuram, Kerala 695016, India
}

Received: 10 June 2016 / Accepted: 27 June 2016 / Published online: 15 July 2016

(C) The Author(s) 2016. This article is published with open access at Springerlink.com

\begin{abstract}
The thermodynamic entropy of an isolated system is given by its von Neumann entropy. Over the last few years, there has been an intense activity to understand the thermodynamic entropy from the principles of quantum mechanics. More specifically, is there a relation between the (von Neumann) entropy of entanglement between a system and some (separate) environment and the thermodynamic entropy? It is difficult to obtain the relation for many body systems, hence, most of the work in the literature has focused on small number systems. In this work, we consider black holes-which are simple yet macroscopic systemsand show that a direct connection could not be made between the entropy of entanglement and the Hawking temperature. In this work, within the adiabatic approximation, we explicitly show that the Hawking temperature is indeed given by the rate of change of the entropy of entanglement across a black hole's horizon with regard to the system energy. This is yet other numerical evidence leading to understanding the key features of black-hole thermodynamics from the viewpoint of quantum information theory.
\end{abstract}

\section{Introduction}

Equilibrium statistical mechanics allows a successful description of the thermodynamic properties of matter [14]. More importantly, it relates entropy, a phenomenological quantity in thermodynamics, to the volume of a certain region in phase space [5]. The laws of thermodynamics are also equally applicable to quantum mechanical systems. A lot of progress has been made recently in studying the cold trap atoms that are largely isolated from surroundings [69]. Furthermore, the availability of Feshbach resonances is shown to be useful to control the strength of interactions, to realize strongly correlated systems, and to drive these

\footnotetext{
a e-mail: santhu@iisertvm.ac.in

be-mail: shanki@iisertvm.ac.in
}

systems between different quantum phases in a controlled manner [10-13]. These experiments have raised the possibility of understanding the emergence of thermodynamics from the principles of quantum mechanics. The fundamental questions that one hopes to answer from these investigations are: How do the macroscopic laws of thermodynamics emerge from the reversible quantum dynamics? How should we understand the thermalization of a closed quantum systems? What are the relations between information, thermodynamics, and quantum mechanics [14-19]? While answering these questions for a many body system is out of sight, some important progress has been made by considering simple lattice systems (see, for instance, Refs. [20-23]). In this work, in an attempt to address some of the above questions, our focus is on another simple, yet, macroscopic system-the black hole.

It has long been conjectured that a black hole's thermodynamic entropy is given by its entropy of entanglement across the horizon [24-30]. However, this has never been directly related to the Hawking temperature [31]. Here we show that:

(i) The Hawking temperature is given by the rate of change of the entropy of entanglement across a black hole's horizon with regard to the system energy.

(ii) The information lost across the horizon is related to the black-hole entropy and the laws of black-hole mechanics emerge from entanglement across the horizon.

The model we consider is complementary to other models that investigate the emergence of thermodynamics [1419]. First, we evaluate the entanglement entropy for a relativistic free scalar fields propagating in the black-hole background, while the simple lattice models that were considered are non-relativistic. Second, quantum entanglement can be unambiguously quantified only for bipartite systems [32,33]. While the bipartite system is an approximation for applications to many body systems, here, the event horizon provides a natural boundary. 
The entanglement of a relativistic free scalar field, as always, is the simplest model to evaluate. However, even for free fields it is difficult to obtain the entanglement entropy. The free fields are Gaussian and these states are entirely characterized by the covariance matrix. It is generally difficult to handle covariance matrices in an infinite-dimensional Hilbert space [33]. There are two ways to calculate the entanglement entropy in the literature. One approach is to use the replica trick which rests on evaluating the partition function on an n-fold cover of the background geometry where a cut is introduced throughout the exterior of the entangling surface $[33,34]$. Second we have a direct approach, where the Hamiltonian of the field is discretized and the reduced density matrix is evaluated in the real space. We adopt this approach as the entanglement entropy may have more symmetries than the Lagrangian of the system [35].

To remove the spurious effects due to the coordinate singularity at the horizon, ${ }^{1}$ we consider a Lemaître coordinate, which is explicitly time-dependent [37]. One of the features that we exploit in our computation is that for a fixed Lemaître time coordinate, the Hamiltonian of the scalar field in Schwarzschild space-time reduces to the scalar field Hamiltonian in flat space-time [28].

The procedure we adopt is the following:

1. We perturbatively evolve the Hamiltonian about the fixed Lemaître time.

2. We obtain the entanglement entropy at different times. We show that at all times, the entanglement entropy satisfies the area law i.e. $S(\epsilon)=C(\epsilon) A$ where $S(\epsilon)$ is the entanglement entropy evaluated at a given Lemaître time $(\epsilon), C(\epsilon)$ is the proportionality constant that depends on $\epsilon$, and $A$ is the area of the black-hole horizon. In other words, the value of the entropy is different at different times.

3. We calculate the change in entropy as a function of $\epsilon$, i.e., $\Delta S / \Delta \epsilon$. Similarly we calculate the change in energy $E(\epsilon)$, i.e., $\Delta E / \Delta \epsilon$.

For several black-hole metrics, we explicitly show that the ratio of the rate of change of energy and the rate of change of entropy is identical to the Hawking temperature.

The outline of the paper is as follows: in Sect. 2, we set up our model Hamiltonian to obtain the entanglement entropy in $(D+2)$-dimensional space-time. Also, we define the entanglement temperature, which has the same structure as in statistical mechanics, that is, the ratio of the change in total energy to the change in entanglement entropy. In Sect. 3 , we numerically show that for different black-hole spacetimes, the divergence free entanglement temperature matches approximately with the Hawking temperature obtained from

${ }^{1}$ In Schwarzschild coordinates, $r>2 M$ needs to be bipartitioned [36]. the general theory of relativity and its Lovelock generalization. This provides strong evidence toward the interpretation of entanglement entropy as the Bekenstein-Hawking entropy. Finally in Sect. 4, we conclude with a discussion to connect our analysis with the eigenstate thermalization hypothesis for closed quantum systems [22].

Throughout this work, the metric signature we adopt is $(+,-,-,-)$ and we set $\hbar=k_{B}=c=1$.

\section{Model and setup}

\subsection{Motivation}

Before we evaluate the entanglement entropy (EE) of a quantum scalar field propagating in a black-hole background, we briefly discuss the motivation for studying the entanglement entropy of a scalar field. Consider the Einstein-Hilbert action with a positive cosmological constant $(|\Lambda|)$ :

$\mathcal{S}_{E H}(\bar{g})=M_{\mathrm{Pl}}^{2} \int \mathrm{d}^{4} x \sqrt{-\bar{g}}[\bar{R}-2|\Lambda|]$

where $\bar{R}$ is the Ricci scalar and $M_{\mathrm{Pl}}$ is the Planck mass. Perturbing the above action w.r.t. the metric $\bar{g}_{\mu \nu}=g_{\mu \nu}+h_{\mu \nu}$, the action up to second order becomes [28]

$$
\begin{aligned}
\mathcal{S}_{E H}(g, h)= & -\frac{M_{\mathrm{Pl}}^{2}}{2} \int d^{4 x} \sqrt{|g|} \\
& \times\left[\nabla_{\alpha} h_{\mu \nu} \nabla^{\alpha} h^{\mu \nu}+|\Lambda| h_{\mu \nu} h^{\mu \nu}\right] .
\end{aligned}
$$

The above action corresponds to a massive $(\Lambda)$ spin-2 field $\left(h_{\mu \nu}\right)$ propagating in the background metric $g_{\mu \nu}$. Rewriting $h_{\mu \nu}=M_{\mathrm{Pl}}^{-1} \epsilon_{\mu \nu} \Phi\left(x^{\mu}\right)$ [where $\epsilon_{\mu \nu}$ is the constant polarization tensor], the above action can be written as

$\mathcal{S}_{E H}(g, h)=-\frac{1}{2} \int \mathrm{d}^{4} x \sqrt{|g|}\left[\partial_{\alpha} \Phi \partial^{\alpha} \Phi+|\Lambda| \Phi^{2}\right]$,

which is the action for the massive scalar field propagating in the background metric $g_{\mu \nu}$. In this work, we consider a massless ( $\Lambda=0$ corresponding to asymptotically flat space-time) scalar field propagating in a $(D+2)$-dimensional spherically symmetric space-time.

\subsection{Model}

The canonical action for the massless, real scalar field $\Phi\left(x^{\mu}\right)$ propagating in $(D+2)$-dimensional space-time is

$\mathcal{S}=\frac{1}{2} \int \mathrm{d}^{D+2} \mathbf{x} \sqrt{-g} g^{\mu \nu} \partial_{\mu} \Phi(\mathbf{x}) \partial_{\nu} \Phi(\mathbf{x})$ 
where $g_{\mu \nu}$ is the spherically symmetric Lemaitre line element [37]:

$\mathrm{d} s^{2}=\mathrm{d} \tau^{2}-(1-f[r(\tau, \xi)]) \mathrm{d} \xi^{2}-r^{2}(\tau, \xi) \mathrm{d} \Omega_{D}^{2}$

where $\tau, \xi$ are the time and radial components in Lemaitre coordinates, respectively, $r$ is the radial distance in Schwarzschild coordinates and $d \Omega_{D}$ is the $D$-dimensional angular line element. In order for the line element (5) to describe a black hole, the space-time must contain a singularity (say at $r=0$ ) and have horizons. We assume that the asymptotically flat space-time contains one non-degenerate event horizon at $r_{h}$. The specific forms of $f(r)$ correspond to different space-times.

The Lemaitre coordinate system has the following interesting properties:

1. The coordinate $\tau$ is time-like all across $0<r<\infty$, similarly $\xi$ is space-like all across $0<r<\infty$.

2. The Lemaître coordinate system does not have coordinate singularity at the horizon.

3. This coordinate system is time-dependent. The test particles at rest relative to the reference system are particles moving freely in the given field [37].

4. The scalar field propagating in this coordinate system is explicitly time-dependent.

The spherical symmetry of the line element (5) allows us to decompose the normal modes of the scalar field as follows:

$\Phi(\mathbf{x})=\sum_{l, m_{i}} \Phi_{l m_{i}}(\tau, \xi) Z_{l m_{i}}\left(\theta, \phi_{i}\right)$,

where $i \in\{1,2, \ldots D-1\}$ and $Z_{l m_{i}}$ 's are the real hyperspherical harmonics. We define the following dimensionless parameters: $\tilde{r}=r / r_{h}, \tilde{\xi}=\xi / r_{h}, \tilde{\tau}=\tau / r_{h}, \tilde{\Phi}_{l m}=$ $r_{h} \Phi_{l m}$. By the substitution of the orthogonal properties of $Z_{l m_{i}}$, the canonical massless scalar field action becomes

$$
\begin{aligned}
\mathcal{S}= & \frac{1}{2} \sum_{l, m_{i}} \int \mathrm{d} \tilde{\tau} \mathrm{d} \tilde{\xi} \tilde{r}^{D}\left[\sqrt{1-f[\tilde{r}]}\left(\partial_{\tilde{\tau}} \tilde{\Phi}_{l m_{i}}\right)^{2}-\frac{1}{\sqrt{1-f[\tilde{r}]}}\right. \\
& \left.\times\left(\partial_{\tilde{\xi}} \tilde{\Phi}_{l m_{i}}\right)^{2}-\sqrt{1-f[\tilde{r}]} \frac{l(l+D-1)}{\tilde{r}^{2}} \tilde{\Phi}_{l m_{i}}^{2}\right] .
\end{aligned}
$$

The above action contains non-linear time-dependent terms through $f(\tilde{r})$. Hence, the Hamiltonian obtained from the above action will have a non-linear time-dependence. While the full non-linear time-dependence is necessary to understand the small size black holes, for large size black holes it is sufficient to linearize the above action by fixing the timeslice and performing an infinitesimal transformation about a particular Lemaître time $\tilde{\tau}$ [38]. More specifically,

$\tilde{\tau} \rightarrow \tilde{\tau}^{\prime}=\tilde{\tau}+\epsilon, \quad \tilde{\xi} \rightarrow \tilde{\xi}^{\prime}=\tilde{\xi}$, $\tilde{r}\left(\tilde{\tau}^{\prime}, \tilde{\xi}^{\prime}\right)=\tilde{r}(\tilde{\tau}+\epsilon, \tilde{\xi})$,

$\tilde{\Phi}_{l m_{i}}(\tilde{\tau}, \tilde{\xi}) \rightarrow \tilde{\Phi}_{l m_{i}}^{\prime}\left(\tilde{\tau}^{\prime}, \tilde{\xi}^{\prime}\right)=\tilde{\Phi}_{l m_{i}}(\tilde{\tau}, \tilde{\xi})$

where $\epsilon$ is the infinitesimal Lemaitre time. The functional expansion of $f(\tilde{r})$ about $\epsilon$ and the following relation between the Lemaître coordinates [37]:

$\tilde{\xi}-\tilde{\tau}=\int \frac{\mathrm{d} \tilde{r}}{\sqrt{1-f[\tilde{r}(\tilde{\tau}, \tilde{\xi})]}}$,

allow us to perform the perturbative expansion in the above action.

After doing the Legendre transformation, the Hamiltonian up to second order in $\epsilon$ is

$H(\epsilon) \simeq H_{0}+\epsilon V_{1}+\epsilon^{2} V_{2}$

where $H_{0}$ is the unperturbed scalar field Hamiltonian in the flat space-time, $V_{1}$ and $V_{2}$ are the perturbed parts of the Hamiltonian (for details, see Appendix A). Physically, the above infinitesimal transformations (10) correspond to perturbatively expanding the scalar field about a particular Lemaître time.

\subsection{Important observations}

The Hamiltonian in Eq. (12) is key equation regarding which we would like to stress the following points: First, in the limit of $\epsilon \rightarrow 0$, the Hamiltonian reduces to that of a free scalar field propagating in flat space-time [28]. In other words, the zeroth order Hamiltonian is identical for all the space-times. Higher order $\epsilon$ terms contain information as regards the global spacetime structure and, more importantly, the horizon properties.

Second, the Lemaitre coordinate is intrinsically timedependent; the $\epsilon$ expansion of the Hamiltonian corresponds to the perturbation about the Lemaitre time. Here, we assume that the Hamiltonian $H$ undergoes adiabatic evolution and the ground state $\Psi_{G S}$ is the instantaneous ground state at all Lemaître times. This assumption is valid for large black holes, as Hawking evaporation is not significant. Also, since the line element is time-asymmetric, the vacuum state is the Unruh vacuum. Evaluation of the entanglement entropy for different values of $\epsilon$ corresponds to different values of Lemaître time. As we will show explicitly in the next section, the entanglement entropy at a given $\epsilon$ satisfies the area law $[S(\epsilon) \propto A]$ and the proportionality constant depends on $\epsilon$, i.e. $S(\epsilon)=C(\epsilon) A$.

Third, it is not possible to obtain a closed form analytic expression for the density matrix (tracing out the quantum degrees of freedom associated with the scalar field inside a spherical region of radius $r_{h}$ ) and hence, we need to resort 
to numerical methods. In order to do so, we take a spatially uniform radial grid, $\left\{r_{j}\right\}$, with $b=r_{j+1}-r_{j}$. We discretize the Hamiltonian $H$ in Eq. (12). The procedure to obtain the entanglement entropy for different $\epsilon$ is similar to the one discussed in Refs. $[25,28]$. In this work, we assume that the quantum state corresponding to the discretized Hamiltonian is the ground state with wave function $\Psi_{G S}\left(x_{1}, \ldots, x_{n} ; y_{1}, \ldots, y_{N-n}\right)$. The reduced density matrix $\rho\left(\mathbf{y}, \mathbf{y}^{\prime}\right)$ is obtained by tracing over the first $n$ of the $N$ oscillators,

$\rho\left(\mathbf{y}, \mathbf{y}^{\prime}\right)=\int\left(\prod_{i=1}^{n} \mathrm{~d} x_{i}\right) \Psi_{G S}\left(x_{1}, . ., x_{n} ; \mathbf{y}\right) \Psi_{G S}^{*}\left(x_{1}, . ., x_{n} ; \mathbf{y}^{\prime}\right)$.

Fourth, in this work, we use the von Neumann entropy

$S(\epsilon)=-\operatorname{Tr}(\rho \log \rho)$

as the measure of entanglement. In analogy with the microcanonical ensemble picture of equilibrium statistical mechanics, the evaluation of the Hamiltonian $H$ at different infinitesimal Lemaitre times, $\epsilon$, corresponds to setting the system at different internal energies. In analogy we define entanglement temperature [39]:

$\frac{1}{T_{E E}}=\frac{\Delta S(\epsilon)}{\Delta E(\epsilon)}=\frac{\text { Slope of } \operatorname{EE}(\Delta S / \Delta \epsilon)}{\text { Slope of energy }(\Delta E / \Delta \epsilon)}$.

The above definition is consistent with the statistical mechanical definition of the temperature. In statistical mechanics, the temperature is obtained by evaluating the change in the entropy and energy w.r.t. the thermodynamic quantities. In our case, the entanglement entropy and the energy depend on the Lemaître time, and we have evaluated the change in the entanglement entropy and energy w.r.t. $\epsilon$. In other words, we calculate the change in the ground state energy (entanglement entropy) for different values of $\epsilon$ and find the ratio of the change in the ground state energy and change in the EE. As we will show in the next section, EE and energy go linearly with $\epsilon$ and, hence, the temperature does not depend on $\epsilon$. While the EE and the energy diverge, their ratio is a non-divergent quantity. To understand this, let us do a dimensional analysis,

$$
\begin{aligned}
& {\left[E_{D+1}\right] \propto N^{D+1} \propto\left(\frac{L}{b}\right)^{D+1}, \quad[S] \propto \frac{A_{D}}{b^{D}}} \\
& \quad \Rightarrow\left[\tilde{T}_{E E}\right] \propto \frac{\left[E_{D+1}\right]}{[S]} \propto N \frac{L^{D}}{A_{D}}, \\
& \text { i.e., } \frac{\left[\tilde{T}_{E E}\right]}{N}=\left[T_{E E}\right] \propto(N / n)^{D} \Rightarrow \text { finite }
\end{aligned}
$$

where $A_{D}$ is the $(D+1)$-dimensional hyper-surface area. In the thermodynamic limit, by setting $L$ finite with $N \rightarrow \infty$ and $b \rightarrow 0, T_{E E}$ in Eq. (16) is finite and independent of $\epsilon$.
For large $N$, we show that, in natural units, the above calculated temperature is identical to the Hawking temperature for the corresponding black hole [31]:

$T_{B H}=\frac{\kappa}{2 \pi}=\left.\frac{1}{4 \pi} \frac{\mathrm{d} f(r)}{\mathrm{d} r}\right|_{r=r_{h}}$.

Fifth, it is important to note that the above entanglement temperature is non-zero only for $f(r) \neq 1$. In the case of a flat space-time, our analysis shows that the entanglement temperature vanishes, and we obtain $T_{E E}$ numerically for different black-hole space-times.

\section{Results and discussions}

The Hamiltonian $H$ in Eq. (12) is mapped to a system of $N$ coupled time independent harmonic oscillators (HOs) with non-periodic boundary conditions. The interaction matrix elements of the Hamiltonian can be found in Ref. [40]. The total internal energy (E) and the entanglement entropy $(S)$ for the ground state of the HOs is computed numerically as a function of $\epsilon$ by using a central difference scheme (see Appendix B). All the computations are done using MATLAB R2012a for the lattice size $N=600,10 \leq n \leq 500$ with a minimum accuracy of $10^{-8}$ and a maximum accuracy of $10^{-12}$.

In the following subsections, we compute $T_{E E}$ numerically for two different black-hole space-times, namely, the four-dimensional Schwarzschild and Reissner-Nordström black holes and show that they match with the Hawking temperature $T_{B H} . T_{E E}$ is calculated by taking the average of entanglement temperature for each $n$ by fixing $N$.

\subsection{Schwarzschild (SBH) black holes}

The four-dimensional Schwarzschild black-hole space-time (put $D=2$ ) in dimensionless units $\tilde{r}$ is given by the line element in Eq. (5) with $f(\tilde{r})$ given by

$f(\tilde{r})=1-\frac{1}{\tilde{r}}$.

In Fig. 1, we have plotted the total energy (in dimensionless units) and EE versus $\epsilon$ for a four-dimensional Schwarzschild space-time. The following points are important to note regarding the numerical results: First for every $\epsilon$, the von Neumann entropy scales approximately as $S \sim\left(r_{h} / b\right)^{2}$. Second, EE and the total energy increase with $\epsilon$.

Using Eq. (15), we evaluate the "entanglement" temperature numerically. In dimensionless units, we get $T_{E E}=$ 0.0793 , which is close to the value of the Hawking temperature 0.079 . However, it is important to note that for different values of $N$, we obtain approximately the same value 

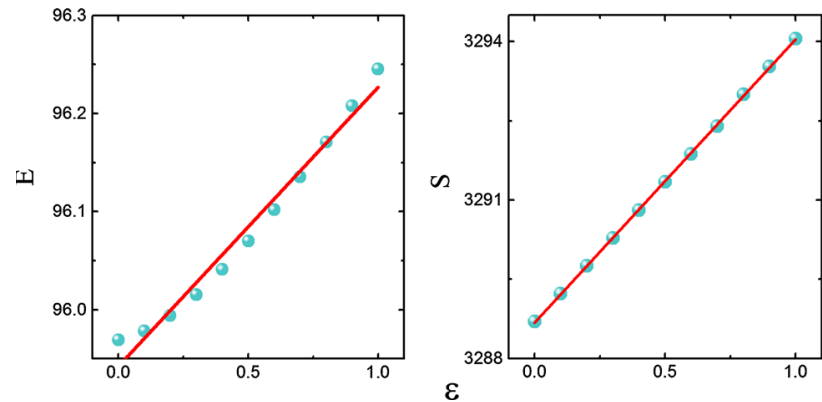

Fig. 1 The plots of total energy (left) and EE (right) as a function of $\epsilon$ for the four-dimensional Schwarzschild black hole. We set $N=600$ and $n=150$. The cyan colored dots are the numerical data and the red line is the best linear fit to the data

Table 1 Entanglement temperature and Hawking temperature (measured in units of $r_{h}$ ) for four-dimensional Schwarzschild and ReissnerNordström black-hole space-times

\begin{tabular}{lll}
\hline Four-dimensional black-hole space-time & $T_{B H}$ & $T_{E E}$ \\
\hline SBH & 0.07958 & 0.07927 \\
RN & & \\
$\quad q=0.1$ & 0.07878 & 0.07836 \\
$q=0.2$ & 0.07639 & 0.07507 \\
$q=0.3$ & 0.07242 & 0.07501 \\
$q=0.4$ & 0.06685 & 0.06659 \\
\hline
\end{tabular}

of the entropy. The results are tabulated; see Table 1 . See Appendix C, for plots of energy and EE for $n=50,80,100$, and 130 .

\subsection{Reissner-Nordström (RN) black holes}

The four-dimensional Reissner-Nordström black hole is given by the line element in Eq. (5), where $f(\tilde{r})$ is

$f(\tilde{r})=1-\frac{2 M / r_{h}}{\tilde{r}}+\frac{\left(Q / r_{h}\right)^{2}}{\tilde{r}^{2}}$.

$Q$ is the charge of the black hole. Note that we have rescaled the radius w.r.t. the outer horizon $\left(r_{h}=M+\sqrt{M^{2}-Q^{2}}\right)$. Choosing $q=Q / r_{h}$, we get

$f(\tilde{r})=1-\frac{\left(1+q^{2}\right)}{\tilde{r}}+\frac{q^{2}}{\tilde{r}^{2}}$

and the black-hole temperature in the unit of $r_{h}$ is $T_{B H}=$ $\left(1-q^{2}\right) / 4 \pi$.

Note that we have evaluated the entanglement temperature by fixing the charge $q$. For a fixed charge $q$, the first law of black-hole mechanics is given by $\mathrm{d} E=(\kappa / 2 \pi) \mathrm{d} A$, where $A$ is the area of the black-hole horizon. The energy and EE for different $q$ values have the same profile, which looks exactly like the previous case and is shown in the middle row in Fig. 2.
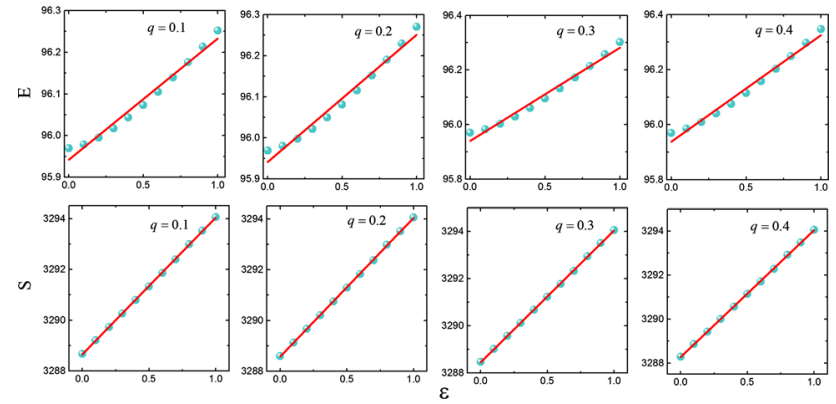

Fig. 2 The plots of total energy (top panel) and EE (bottom panel) as a function of $\epsilon$ for different $q \mathrm{~s}$ in four-dimensional Reissner-Nordström black hole. For these, we set $N=600$ and $n=150$. The cyan colored dots are the numerical data and the red line is the best linear fit to the data

See Appendix C for plots for other values of $n$. As given in Table 1, $T_{E E}$ matches with the Hawking temperature.

\section{Conclusions and outlook}

In this work, we have given another proof that fourdimensional black-hole entropy can be associated to the entropy of entanglement across the horizon by explicitly deriving the entanglement temperature. The entanglement temperature is given by the rate of change of the entropy of entanglement across a black hole's horizon with regard to the system energy. Our new result sheds light on the interpretation of temperature as regards entanglement as the Hawking temperature; one more step to understanding the black-hole thermodynamics in the field of quantum information theory.

Some of the key features of our analysis are: First, while entanglement and energy diverge in the limit of $b \rightarrow 0$, the entanglement temperature is a finite quantity. Second, the entanglement temperature vanishes for the flat spacetime. While the evaluation of the entanglement entropy does not distinguish between the black-hole space-time and flat space-time, the entanglement temperature distinguishes the two space-times.

Our analysis also shows that the entanglement entropy satisfies all the properties of the black-hole entropy. First, like the black-hole entropy, the entanglement entropy increases and never decreases. Second, the entanglement entropy and the temperature satisfies the first law of black-hole mechanics $\mathrm{d} E=T_{E E} \mathrm{~d} S$. We have shown this explicitly for a Schwarzschild black hole and for a Reissner-Norstrom black hole.

It is quite remarkable that in higher-dimensional spacetime the Rényi entropy provides a convergent alternative to the measure of entanglement [30]; however, the entanglement temperature will depend on the Rényi parameter. While a physical understanding of the Rényi parameter has emerged 
[41], it is still not clear how to fix the Rényi parameter from first principles [42].

Our analysis throws some light on the emergent gravity paradigm [43-46] where gravity is not viewed as a fundamental force. Here we have shown that the information lost across the horizon is related to the black-hole entropy and the laws of black-hole mechanics emerge from the entanglement across the horizon. Since general relativity reduces gravity to an effect of the curvature of the space-time, it is thought that the microscopic constituents would be the atoms of the space-time itself. Our analysis shows that entanglement across horizons can be used as building blocks of space-time $[47,48]$.

One of the unsettling questions in theoretical physics is whether due to Hawking temperature the black hole has performed a non-unitary transformation on the state of the system, a.k.a. the information loss problem. Our analysis here does not address this question for two reasons: (1) Here, we have fixed the radius of the horizon at all times and evaluated the change in the entropy while to address the information loss we need to consider a changing horizon radius. (2) Here, we have used a perturbative Hamiltonian, and, hence, this analysis fails as the black-hole size shrinks to half its size [49]. We hope to report on this feature in the future.

While the unitary quantum time-evolution is reversible and retains all information as regards the initial state, we have shown that the restriction of the degrees of freedom outside the event horizon at all times leads to a temperature analogous to the Hawking temperature. Our analysis may have relevance to the eigenstate thermalization hypothesis [20-23], which we plan to explore.

Acknowledgments The authors wish to thank A. P. Balachandran, Charles Bennett, Samuel Braunstein, Saurya Das, and Jens Eisert for discussions and comments. Also, we would like to thank the anonymous referee for the useful comments. All numerical computations were done at the fast computing clusters at IISER-TVM. The work is supported by Max Planck-India Partner Group on Gravity and Cosmology. SSK acknowledges the financial support of the CSIR, Govt. of India through Senior Research Fellowship. SS is partially supported by Ramanujan Fellowship of DST, India.

Open Access This article is distributed under the terms of the Creative Commons Attribution 4.0 International License (http://creativecomm ons.org/licenses/by/4.0/), which permits unrestricted use, distribution, and reproduction in any medium, provided you give appropriate credit to the original author(s) and the source, provide a link to the Creative Commons license, and indicate if changes were made.

Funded by SCOAP ${ }^{3}$.

\section{Appendix A: Calculation of scalar field Hamiltonian in Lemaître coordinate}

In this appendix, we give details of the derivation of the Hamiltonian $(\mathrm{H})$ up to second order in $\epsilon$. Using the orthogo- nal properties of the real spherical harmonics $Z_{l m_{i}}$, the scalar field action reduces to

$$
\begin{aligned}
\mathcal{S}= & \frac{1}{2} \sum_{l, m_{i}} \int \mathrm{d} \tilde{\tau} \mathrm{d} \tilde{\xi} \tilde{r}^{D}\left[\sqrt{1-f[\tilde{r}]}\left(\partial_{\tilde{\tau}} \tilde{\Phi}_{l m_{i}}\right)^{2}-\frac{\left(\partial_{\tilde{\xi}} \tilde{\Phi}_{l m_{i}}\right)^{2}}{\sqrt{1-f[\tilde{r}]}}\right. \\
& \left.-\sqrt{1-f[\tilde{r}]} \frac{l(l+D-1)}{\tilde{r}^{2}} \tilde{\Phi}_{l m_{i}}^{2}\right]
\end{aligned}
$$

where $\tilde{r}=r / r_{h}, \tilde{\xi}=\xi / r_{h}, \tilde{\tau}=\tau / r_{h}, \tilde{\Phi}_{l m}=r_{h} \Phi_{l m}$ are dimensionless.

We perform the following infinitesimal transformation [38] in the above resultant action:

$\tilde{\tau} \rightarrow \tilde{\tau}^{\prime}=\tilde{\tau}+\epsilon, \tilde{\xi} \rightarrow \tilde{\xi}^{\prime}=\tilde{\xi}$,

$\tilde{\Phi}_{l m_{i}}(\tilde{\tau}, \tilde{\xi}) \rightarrow \tilde{\Phi}_{l m_{i}}^{\prime}\left(\tilde{\tau}^{\prime}, \tilde{\xi}^{\prime}\right)=\tilde{\Phi}_{l m_{i}}(\tilde{\tau}, \tilde{\xi})$,

$\tilde{r}\left(\tilde{\tau}^{\prime}, \tilde{\xi}^{\prime}\right)=\tilde{r}(\tilde{\tau}+\epsilon, \tilde{\xi})$.

The action in Eq. A.1 becomes

$$
\begin{aligned}
\mathcal{S} \simeq & \frac{1}{2} \sum_{l, m_{i}} \int \mathrm{d} \tilde{\tau} \mathrm{d} \tilde{\xi}\left(\tilde{r}+\epsilon h_{1}+\epsilon^{2} h_{2} / 2\right)^{D} \\
& \times\left[\left(1-f-\epsilon h_{1} \frac{\partial f}{\partial \tilde{r}}-\frac{\epsilon^{2}}{2}\left[h_{2} \frac{\partial f}{\partial \tilde{r}}+h_{1}^{2} \frac{\partial^{2} f}{\partial \tilde{r}^{2}}\right]\right)^{1 / 2}\left(\partial_{\tilde{\tau}} \tilde{\Phi}_{l m_{i}}\right)^{2}\right. \\
& -\left(1-f-\epsilon h_{1} \frac{\partial f}{\partial \tilde{r}}-\frac{\epsilon^{2}}{2}\left[h_{2} \frac{\partial f}{\partial \tilde{r}}+h_{1}^{2} \frac{\partial^{2} f}{\partial \tilde{r}^{2}}\right]\right)^{-1 / 2}\left(\partial_{\tilde{\xi}} \tilde{\Phi}_{l m_{i}}\right)^{2} \\
& -\frac{l(l+D-1)}{\left(\tilde{r}+\epsilon h_{1}+\epsilon^{2} h_{2} / 2\right)^{2}} \\
& \left.\times\left(1-f-\epsilon h_{1} \frac{\partial f}{\partial \tilde{r}}-\frac{\epsilon^{2}}{2}\left[h_{2} \frac{\partial f}{\partial \tilde{r}}+h_{1}^{2} \frac{\partial^{2} f}{\partial \tilde{r}^{2}}\right]\right)^{1 / 2} \tilde{\Phi}_{l m_{i}}^{2}\right]
\end{aligned}
$$

where $h_{1}=\frac{\partial \tilde{r}}{\partial \tilde{\tau}}$ and $h_{2}=\frac{\partial^{2} \tilde{r}}{\partial \tilde{\tau}^{2}}$.

Using the relation between the Lemaitre coordinates [37]

$\xi-\tau=\int \frac{\mathrm{d} r}{\sqrt{1-f[r(\tau, \xi)]}}$

gives the following expression:

$h_{1}=-\sqrt{1-f}, \quad h_{2}=\frac{-1}{2} \frac{\partial f}{\partial \tilde{r}}, \quad \frac{\mathrm{d} \tilde{\xi}}{\mathrm{d} \tilde{r}} \mid \operatorname{con} \cdot \tilde{\tau}=\frac{1}{\sqrt{1-f}}$

The Hamiltonian $(H)$ corresponding to the above Lagrangian is 


$$
\begin{aligned}
& H \simeq \frac{1}{2} \sum_{l, m_{i}} \int \mathrm{d} \tilde{\xi}\left[\tilde{\Pi}_{l m_{i}}^{2}+\frac{g_{1}^{D}}{g_{2}}\left(\partial_{\tilde{\xi}} \frac{\tilde{\chi}_{l m_{i}}}{g_{1}^{D / 2} \sqrt{g_{2}}}\right)^{2}\right. \\
& H_{0}=\frac{1}{2} \sum_{l, m_{i}} \int_{\tilde{\tau}}^{\infty} \mathrm{d} \tilde{r}\left[\pi_{l m_{i}}^{2}+\tilde{r}^{D}\left[\partial_{\tilde{r}} \frac{\sigma_{l m_{i}}}{\tilde{r}^{D / 2}}\right]^{2}\right. \\
& \left.+\frac{l(l+D-1)}{g_{1}^{2}} \tilde{\chi}_{l m_{i}}^{2}\right] \\
& \left.+\frac{l(l+D-1)}{\tilde{r}^{2}} \sigma_{l m_{i}}^{2}\right],
\end{aligned}
$$

where

$$
\begin{aligned}
& g_{1}=\tilde{r}+\epsilon h_{1}+\epsilon^{2} h_{2} / 2, \\
& g_{2}=\sqrt{1-f-\epsilon h_{1} \frac{\partial f}{\partial \tilde{r}}-\frac{\epsilon^{2}}{2}\left[h_{2} \frac{\partial f}{\partial \tilde{r}}+h_{1}^{2} \frac{\partial^{2} f}{\partial \tilde{r}^{2}}\right]}, \\
& \quad \tilde{\chi}_{l m_{i}}=g_{1}^{D / 2} \sqrt{g_{2}} \tilde{\Phi}_{l m_{i}}
\end{aligned}
$$

and $\tilde{\Pi}_{l m_{i}}$ is the canonical conjugate momenta corresponding to the field $\tilde{\chi}_{l m_{i}}$.

Upon quantization, $\tilde{\Pi}_{l m_{i}}$ and $\tilde{\chi}_{l m_{i}}$ satisfy the usual canonical commutation relation:

$$
\left[\tilde{\chi}_{l m_{i}}(\tilde{\xi}, \tilde{\tau}), \tilde{\Pi}_{l^{\prime} m_{i}^{\prime}}\left(\tilde{\xi}^{\prime}, \tilde{\tau}\right)\right]=i \delta_{l l^{\prime}} \delta_{m_{i} m_{i}^{\prime}} \delta\left(\tilde{\xi}-\tilde{\xi}^{\prime}\right) .
$$

Using relations (A.7) and expanding the Hamiltonian up to second order in $\epsilon$, we get

$$
\begin{aligned}
H & \simeq \frac{1}{2} \sum_{l, m_{i}} \int_{\tilde{\tau}}^{\infty} \mathrm{d} \tilde{r}\left[\pi_{l m_{i}}^{2}+\tilde{r}^{D} \frac{\left(1-\epsilon H_{1}-\epsilon^{2} H_{2}\right)^{D}}{\left(1+\epsilon H_{3}-\epsilon^{2} H_{4}\right)^{1 / 2}}\right. \\
& \times\left[\frac{\sigma_{l m_{i}}}{\left.\partial_{\tilde{r}} \frac{(\text { A. } 13)}{\tilde{r}^{D / 2}\left(1-\epsilon H_{1}-\epsilon^{2} H_{2}\right)^{D / 2}\left(1+\epsilon H_{3}-\epsilon^{2} H_{4}\right)^{1 / 4}}\right]^{2}}\right. \\
& \left.+\frac{l(l+D-1)}{\tilde{r}^{2}\left(1-\epsilon H_{1}-\epsilon^{2} H_{2}\right)^{2}} \sigma_{l m_{i}}^{2}\right] .
\end{aligned}
$$

The Hamiltonian in Eq. (A.13) is of the form

$$
H \simeq H_{0}+\epsilon V_{1}+\epsilon^{2} V_{2}
$$

where $H_{0}$ is the unperturbed scalar field Hamiltonian in the flat space-time, $V_{1}$ and $V_{2}$ are the perturbed parts of the Hamiltonian given by

$$
\begin{aligned}
V_{2}= & \frac{1}{2} \sum_{l, m_{i}} \int_{\tilde{\tau}}^{\infty} \mathrm{d} \tilde{r}\left[\left(H_{3}^{2}+H_{4}\right) \sigma_{l m_{i}}^{\prime 2}\right. \\
& +\left(-\frac{D H_{3}^{2}}{\tilde{r}}-\frac{D H_{4}}{\tilde{r}}+D H_{1} H_{1}^{\prime}-D H_{3} H_{1}^{\prime}+D H_{2}^{\prime}\right. \\
& \left.+H_{3} H_{3}^{\prime}+\frac{1}{2} H_{4}^{\prime}\right) \sigma_{l m_{i}}^{\prime} \sigma_{l m_{i}} \\
& +\left(\frac{l(l+D-1)\left(3 H_{1}^{2}+2 H_{2}\right)}{\tilde{r}^{2}}+\frac{D^{2} H_{3}^{2}}{4 \tilde{r}^{2}}+\frac{D^{2} H_{4}}{4 \tilde{r}^{2}}\right. \\
& -\frac{D^{2} H_{1} H_{1}^{\prime}}{2 \tilde{r}}+\frac{D^{2} H_{3} H_{1}^{\prime}}{2 \tilde{r}}+\frac{1}{4} D^{2} H_{1}^{\prime 2}-\frac{D^{2} H_{2}^{\prime}}{2 \tilde{r}} \\
& \left.\left.-\frac{D H_{3} H_{3}^{\prime}}{2 \tilde{r}}-\frac{1}{4} D H_{1}^{\prime} H_{3}^{\prime}+\frac{1}{16} H_{3}^{\prime 2}-\frac{d H_{4}^{\prime}}{4 \tilde{r}}\right) \sigma_{l m_{i}}^{2}\right],
\end{aligned}
$$

where

$H_{1}=\frac{\sqrt{1-f}}{\tilde{r}}, \quad H_{2}=\frac{1}{4 \tilde{r}} \frac{\partial f}{\partial \tilde{r}}, \quad H_{3}=\frac{1}{\sqrt{1-f}} \frac{\partial f}{\partial \tilde{r}}$,

$H_{4}=\frac{-1}{4(1-f)}\left(\frac{\partial f}{\partial \tilde{r}}\right)^{2}+\frac{1}{2} \frac{\partial^{2} f}{\partial \tilde{r}^{2}}$,

and the redefined field operators are

$\tilde{\Pi}_{l m_{i}}=\frac{\pi_{l m_{i}}}{(1-f)^{1 / 4}} \quad$ and $\quad \tilde{\chi}_{l m_{i}}=\frac{\sigma_{l m_{i}}}{(1-f)^{1 / 4}}$,

such that they satisfy the following canonical commutation relation:

$\left[\pi_{l m_{i}}(\tilde{r}, \tilde{\tau}), \sigma_{l^{\prime} m_{i}^{\prime}}\left(\tilde{r}^{\prime}, \tilde{\tau}\right)\right]=i \delta_{l l^{\prime}} \delta_{m_{i} m_{i}^{\prime}} \delta\left(\tilde{r}-\tilde{r}^{\prime}\right)$.

The Hamiltonian $H$ in Eq. (A.14) is mapped to a system of $N$ coupled time independent harmonic oscillators (HO) with non-periodic boundary conditions. The interaction matrix elements of the Hamiltonian can be found in Ref. [40]. The total internal energy $(E)$ and the entanglement entropy $(S)$ for the ground state of the HO's is computed numerically as a function of $\epsilon$ by using a central difference scheme. 


\section{Appendix B: Central difference discretization}

Central difference discretization is one of the effective methods for finding the approximate value for the derivative of a function in the neighborhood of any discrete point, $x_{i}=x_{0}+i h$, with unit steps of $h$. The Taylor expansions of the function about the point $x_{0}$ in the forward and backward difference scheme are given, respectively, by

$$
\begin{aligned}
& f(x+h)=f(x)+\frac{h f^{\prime}(x)}{1 !}+\frac{h^{2} f^{\prime \prime}(x)}{2 !}+\cdots, \\
& f(x-h)=f(x)-\frac{h f^{\prime}(x)}{1 !}+\frac{h^{2} f^{\prime \prime}(x)}{2 !}-\cdots,
\end{aligned}
$$

which implies

$$
\begin{aligned}
f^{\prime}(x) & =\frac{f(x+h)-f(x-h)}{2 h}+O\left(h^{2}\right), \\
f^{\prime \prime}(x) & =\frac{f(x+h)-2 f(x)+f(x-h)}{h^{2}}+O\left(h^{2}\right), \\
f^{\prime \prime \prime}(x) & =\frac{f(x+2 h)-2 f(x+h)+2 f(x-h)+f(x-2 h)}{2 h^{3}} \\
+ & O\left(h^{2}\right) .
\end{aligned}
$$

\section{Appendix C: Plots of internal energy and EE as a function of $\epsilon$ for different black-hole space-times}

In this section of the appendix, we give plots of EE for different black-hole space-times (Figs. 3, 4, 5).
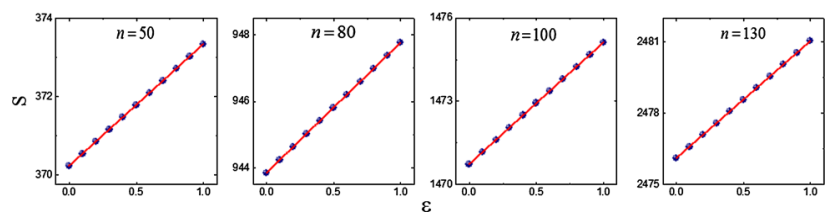

Fig. 3 Plots of the EE as a function of $\epsilon$ for the four-dimensional Schwarzschild black hole with $N=300, n=50,80,100$, and 130, respectively. The blue dots are the numerical data and the red line is the best linear fit to the data
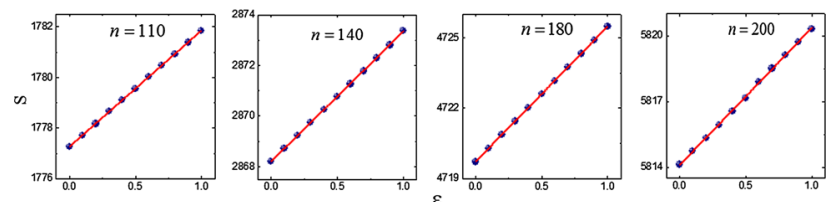

Fig. 4 Plots of the EE as a function of $\epsilon$ for the four-dimensional Schwarzschild black hole with $N=400, n=110,140,180$, and 200, respectively. The blue dots are the numerical data and the red line is the best linear fit to the data

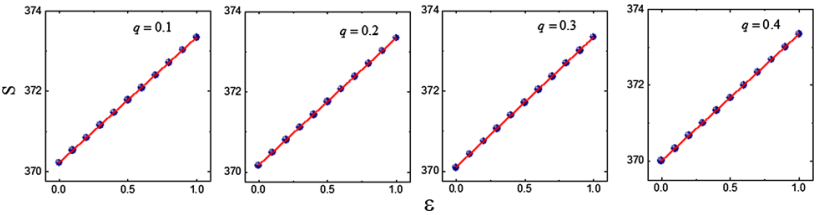

Fig. 5 Plots of the EE of four-dimensional R-N black hole in terms of $\epsilon$ for different $q$ 's with $N=300$, and $n=50$. The blue dots are the numerical data and the red line is the best linear fit to the data

\section{References}

1. G.D. Birkhoff, Proof of a recurrence theorem for strongly transitive systems. Proc. Natl. Acad. Sci. 17, 650-655 (1931). doi:10.1073/ pnas.17.12.650

2. J.V. Neumann, Proof of the quasi-ergodic hypothesis. Proc. Natl. Acad. Sci. 18(1), 70-82 (1932). doi:10.1073/pnas.18.1.70

3. L. Boltzmann, Lectures on Gas Theory (Dover Books on Physics, Dover Publications, New York, 2011)

4. L.D. Landau, E.M. Lifshitz, Statistical Physics, Volume Vof Course of Theoretical Physics, 3rd edn. (Elsevier, Amsterdam, 1980)

5. A. Wehrl, General properties of entropy. Rev. Mod. Phys. 50(2), 221-260 (1978). doi:10.1103/RevModPhys.50.221

6. T. Kinoshita, T. Wenger, D.S. Weiss, A quantum Newton's cradle. Nature 440(7086), 900-903 (2006). doi:10.1038/nature04693

7. J. Esteve, C. Gross, A. Weller, S. Giovanazzi, M.K. Oberthaler, Squeezing and entanglement in a Bose-Einstein condensate. Nature 455(7217), 1216-1219 (2008). doi:10.1038/nature07332

8. D.A. Smith, M. Gring, T. Langen, M. Kuhnert, B. Rauer, R. Geiger, T. Kitagawa, I. Mazets, E. Demler, J. Schmiedmayer, Prethermalization revealed by the relaxation dynamics of full distribution functions. N. J. Phys. 15(7), 075011 (2013). doi:10.1088/ 1367-2630/15/7/075011

9. V.I. Yukalov, Bose-einstein condensation and gauge symmetry breaking. Laser Phys. Lett. 4(9), 632 (2007). doi:10.1002/lapl. 200710029

10. A. Osterloh, L. Amico, G. Falci, R. Fazio, Scaling of entanglement close to a quantum phase transition. Nature 416, 608 (2002). doi: 10. 1038/416608a

11. L.-A. Wu, M.S. Sarandy, D.A. Lidar, Quantum phase transitions and bipartite entanglement. Phys. Rev. Lett. 93, 250-404 (2004). doi:10.1103/PhysRevLett.93.250404

12. J. von Stecher, E. Demler, M.D. Lukin, A.M. Rey, Probing interaction-induced ferromagnetism in optical superlattices. N. J. Phys. 12(5), 055009 (2010). doi:10.1088/1367-2630/12/5/055009

13. J. Dinerman, L.F. Santos, Manipulation of the dynamics of manybody systems via quantum control methods. N. J. Phys. 12(5), 055025 (2010). doi:10.1088/1367-2630/12/5/055025

14. L. Seth, Quantum thermodynamics: excuse our ignorance. Nat. Phys. 2(11), 727-728 (2006). doi:10.1038/nphys456

15. F.G.S.L. Brandao, M.B. Plenio, Entanglement theory and the second law of thermodynamics. Nat. Phys. 4(11), 873-877 (2008). doi:10.1038/nphys1100

16. M. Horodecki, Quantum entanglement: Reversible path to thermodynamics. Nat. Phys. 4(11), 833-834 (2008). doi:10.1038/ nphys 1123

17. S. Popescu, D. Rohrlich, Thermodynamics and the measure of entanglement. Phys. Rev. A 56, R3319-R3321 (1997). doi:10. 1103/PhysRevA.56.R3319

18. V. Vedral, M.B. Plenio, Entanglement measures and purification procedures. Phys. Rev. A 57, 1619-1633 (1998). doi:10.1103/ PhysRevA.57.1619 
19. M.B. Plenio, V. Vedral, Teleportation, entanglement and thermodynamics in the quantum world. Contemp. Phys. 39(6), 431-446 (1998). doi:10.1080/001075198181766

20. M. Srednicki, Chaos and quantum thermalization. Phys. Rev. E 50, 888-901 (1994). doi:10.1103/PhysRevE.50.888

21. M. Rigol, V. Dunjko, M. Olshanii, Thermalization and its mechanism for generic isolated quantum systems. Nature 452(7189), 854-858 (2008). doi:10.1038/nature06838

22. M. Rigol, M. Srednicki, Alternatives to eigenstate thermalization. Phys. Rev. Lett. 108, 110601 (2012). doi:10.1103/PhysRevLett. 108.110601

23. R. Nandkishore, D.A. Huse, Many-body localization and thermalization in quantum statistical mechanics. Annu. Rev. Condens. Matter Phys. 6(1), 15-38 (2015). doi:10.1146/ annurev-conmatphys-031214-014726

24. L. Bombelli, R.K. Koul, J. Lee, R.D. Sorkin, Quantum source of entropy for black holes. Phys. Rev. D 34, 373-383 (1986). doi:10. 1103/PhysRevD.34.373

25. M. Srednicki, Entropy and area. Phys. Rev. Lett. 71, 666-669 (1993). doi:10.1103/PhysRevLett.71.666

26. J. Eisert, M. Cramer, Single-copy entanglement in critical quantum spin chains. Phys. Rev. A 72, 042112 (2005). doi:10.1103/ PhysRevA.72.042112

27. S. Das, S. Shankaranarayanan, How robust is the entanglement entropy-area relation? Phys. Rev. D 73, 121701 (2006). doi:10. 1103/PhysRevD.73.121701

28. S. Das, S. Shankaranarayanan, S. Sur, Black hole entropy from entanglement: a review, in Horizons in World Physics, vol. 268, ed. by M. Everett, L. Pedroza (Nova Science Publishers, New York, 2009), p. 211

29. S.N. Solodukhin, Entanglement entropy of black holes. Living Rev. Relativ. 14, 1104-3712 (2011). doi:10.12942/lrr-2011-8

30. S.L. Braunstein, S. Das, S. Shankaranarayanan, Entanglement entropy in all dimensions. J. High Energy Phys. 2013(7), 1-9 (2013). doi:10.1007/JHEP07(2013)130

31. S.W. Hawking, Particle creation by black holes. Commun. Math. Phys. 43, 199-220 (1975). doi:10.1007/BF02345020

32. R. Horodecki, P. Horodecki, M. Horodecki, K. Horodecki, Quantum entanglement. Rev. Mod. Phys. 81, 865-942 (2009). doi:10. 1103/RevModPhys.81.865

33. J. Eisert, M. Cramer, M.B. Plenio, Colloquium: area laws for the entanglement entropy. Rev. Mod. Phys. 82, 277-306 (2010). doi:10.1103/RevModPhys.82.277

34. P. Calabrese, J.L. Cardy, Entanglement entropy and quantum field theorydoi. J. Stat. Mech. Theory Exp. 0406, P06002 (2004). doi: 10. 1088/1742-5468/2004/06/P06002
35. K. Mallayya, R. Tibrewala, S. Shankaranarayanan, T. Padmanabhan, Zero modes and divergence of entanglement entropy. Phys. Rev. D 90, 044058 (2014). doi:10.1103/PhysRevD.90.044058

36. S. Mukohyama, M. Seriu, H. Kodama, Thermodynamics of entanglement in Schwarzschild spacetime. Phys. Rev. D 58, 064001 (1998). doi:10.1103/PhysRevD.58.064001

37. L.D. Landau, E.M. Lifshitz, The Classical Theory of Fields Volume II of Course of Theoretical Physics, 4th edn. (ButterworthHeinemann, Oxford, 1975)

38. D.J. Toms, The Schwinger action principle and effective action (Cambridge Monographs on Mathematical Physics. Cambridge Univ. Press, Cambridge, 2007)

39. H. Sakaguchi, Renyi entropy and statistical mechanics. Prog. Theor. Phys. 81(4), 732-737 (1989). doi:10.1143/PTP.81.732

40. Click on the following 'Dropbox' link for more details on MATLAB codes that use for the numerical study reported in the paper. https://www.dropbox.com/sh/wxmq02qxg6m3pbm/ AABEE-k4MMow7FJj_JHgfrV_a?dl=0

41. J.C. Baez, Rényi entropy and free energy. arXiv:1102.2098 [quant$\mathrm{ph}]$

42. Work under progress

43. A.D. Sakharov, Vacuum quantum fluctuations in curved space and the theory of gravitation. Gen. Relativ. Gravit. 32(2), 365-367 (2000). doi:10.1023/A:1001947813563

44. T. Jacobson, Thermodynamics of spacetime: the Einstein equation of state. Phys. Rev. Lett. 75, 1260-1263 (1995). doi:10.1103/ PhysRevLett.75.1260

45. T. Padmanabhan, Thermodynamical aspects of gravity: new insights. Rep. Prog. Phys. 73(4), 046901 (2010). doi:10.1088/ 0034-4885/73/4/046901

46. E. Verlinde, On the origin of gravity and the laws of newton. J. High Energy Phys. 2011(4), 1-27 (2011). doi:10.1007/ JHEP04(2011)029

47. M. Van Raamsdonk, Building up spacetime with quantum entanglement. Gen. Relativ. Gravit. 42(10), 2323-2329 (2010). doi:10. 1007/s10714-010-1034-0

48. M. Van Raamsdonk, Building up spacetime with quantum entanglement. Int. J. Modern Phys. D 19(14), 2429-2435 (2010). doi:10. 1142/S0218271810018529

49. A. Almheiri, D. Marolf, J. Polchinski, J. Sully, Black holes: complementarity or firewalls? J. High Energy Phys. 2013(2), 1-20 (2013). doi:10.1007/JHEP02(2013)062 